\title{
Linking evolution across scales
}

\author{
A full picture of biodiversity across space and time requires macro- and microevolutionary approaches.
}

T he remarkable diversity of life on Earth is a central and unifying theme in ecology and evolution. Understanding how biodiversity is generated and how it varies across space and time requires approaches from both macroand microevolutionary perspectives. For example, we can use the fossil record in combination with molecular phylogenies and modelling to reconstruct patterns of biological variation and determine the mechanisms responsible for generating species diversity over geological timescales. But we also need to understand the mechanisms that promote species formation and persistence at an ecological timescale, including the genomic architecture of traits that contribute to population divergence, the eco-evolutionary forces that act on those traits, and the molecular genetic details and evolutionary forces that promote reproductive isolation. Ultimately, we need to link macroevolutionary patterns to microevolutionary processes - one of the big challenges of biodiversity research. Nature Ecology \& Evolution is interested in the full spectrum of research that uses evolutionary principles to understand biodiversity, and here we highlight some recent studies published in our journal.

One striking macroevolutionary pattern is how some groups are orders of magnitude more diverse than others. Substantial variation in diversification rates across taxa, as well as temporal variation of diversification rates within a group, are well documented by fossil time-series and phylogenetic trees. While iconic groups such as the Darwin finches or Hawaiian silverswords represent rapid and extensive evolutionary radiations, small but frequent changes in extinction and speciation rates are likely to occur across species due to biotic and abiotic factors. Using a new phylogenetic method to determine species-specific diversification rates across avian phylogenies, Maliet et al. (Nat. Ecol. Evol. 3, 1086-1092; 2019) found small but frequent changes in diversification rates during the avian radiation.

Beyond describing patterns of diversification over space and time, macroevolutionary researchers seek to understand the biotic and abiotic mechanisms behind them. For example, combining fossil records with molecular phylogenies of marine diatoms, Lewitus et al. (Nat. Ecol. Evol. 2, 1715-1723; 2018) show an important effect of variation in carbon dioxide partial pressure on early diatom diversification, whereas subsequent diversification was mostly affected by sea level, silica availability and competition with other planktonic groups.

Macroevolutionary patterns also have strong geographical signatures, most notably the latitudinal diversity gradient, where the number of species increases from the poles to the Equator in both marine and terrestrial habitats. This pattern can be explained just by invoking different rates of speciation, extinction and dispersal across the globe, yet it is unclear what mechanisms control such differential rates. In this issue, Saupe et al. use simulations to show that spatiotemporal climatic changes recapitulate the patterns of vertebrate biodiversity as a function of speciation, extinction and dispersal alone. In an accompanying News \& Views, Tomasovych discusses the importance of spatio-temporal models of species distributions coupled with long-term variability in climate.

Adaptive radiations are often associated with the evolution of novel traits that enable species to explore new ecological niches. For example, the adaptive radiation of Antarctic notothenioid fishes following the cooling of the Antarctic was preceded by rapid genomic evolution and reduced ossification and buoyancy, which were selected upon once new niches were available in the polar sea (Daane et al. Nat. Ecol. Evol. 3, 1102-1109; 2019).

The disparate patterns of evolution that we observe across the tree of life over geological timescales ought to result from processes that occur within and between populations at ecological timescales. Speciation rates might vary among taxa due to genetic and ecological mechanisms that control population divergence, reproductive isolation and/ or species persistence. In recent years, genome analysis of many incipient species has uncovered genomic mechanisms and evolutionary forces promoting species origination and maintenance. For example, coupling between vision and colour genes promotes divergence in hamlets, a group of Caribbean reef fishes that differ in colour pattern and are reproductively isolated via assortative mating (Hench et al. Nat. Ecol. Evol. 3, 657-667; 2019). In the Lake Malawi haplochromine cichlid radiation, adaptive introgression and independent selection on visual and oxygen transport genes promoted rapid diversification of deepwater species (Malinsky et al. Nat. Ecol. Evol. 2, 1940-1955; 2018). Mounting genomic data have also revealed the contribution of hybridization to biodiversity. Early this year, Taylor and Larson (Nat. Ecol. Evol. 3, 170-177; 2019) reviewed recent work that shows how hybridization contributes to natural diversity. While evidence for hybridization itself leading to reproductive isolation between the hybrid species and its parental taxa is scarce, the contribution of ancient hybridization to mammalian evolutionary history is well established and there is good evidence for adaptive introgression in a handful of systems such as genes involved in warfarin resistance from Mus spretus to M. musculus domesticus, or two genomic regions that control mimicry patterns between three species of Heliconius butterflies.

At an even finer scale, researchers look for genes that affect reproductive isolation, including those responsible for mating discrimination or hybrid sterility and inviability. Loci underlying genetic incompatibilities have been identified in several model organisms including yeast, flies, mice and plants. Nevertheless, understanding the evolutionary forces that cause the spread of alleles that lead to hybrid dysfunction - mutation and genetic drift, natural selection, or intragenomic conflict - remains an important goal. We look forward to seeing further steps towards a comprehensive view of the temporal and geographical patterns of biodiversity and approaches that seek to bridge the macroand microevolutionary scales.

Published online: 26 September 2019 https://doi.org/10.1038/s41559-019-1001-4 